\title{
DEFECTIVE INTERFERING PARTICLES OF CORONAVIRUS
}

\author{
Shinji Makino ${ }^{1}$, Kosaku Fujiwara ${ }^{2}$ and Michael M.C. Lai ${ }^{1}$ \\ ${ }^{1}$ Department of Microbiology, University of Southern California \\ School of Medicine, Los Angeles, CA 90033 and \\ ${ }^{2}$ Department of Animal Pathology, Institute of Medical Science \\ University of Tokyo, Tokyo 108
}

\section{INTRODUCTION}

Defective interfering (DI) particles are viral deletion mutants, which cannot replicate by themselves and require homologous standard viruses to provide helper functions for their replication. DI particles also interfere with the replication of helper virus. Many studies have suggested a role for DI particles in evolution, persistent infection and pathogenesis of various viruses. Although coronaviruses readily establish persistent infection in vitro and in vivo, there have been no reports of isolation of coronavirus $\overline{\mathrm{DI}}$ particles during persistent infection. We have, however, detected the generation of coronavirus DI particles during high-multiplicity passages of the JHM strain of MHV in tissue culture (Makino et al., 1984a). These DI particles contain a single-stranded RNA genome of roughly $5.2 \times 10^{6}$ molecular weight ${ }_{6}$ which is slightly smaller than the genome of the standard virus (M.W. $5.4 \times 10^{6}$ ). Oligonucleotide fingerprinting studies showed that the RNA of JHM DI is missing several large RNase $T_{1}-$ resistant oligonucleotides, which represent several different regions on the standard viral genome (Makino et al., 1984a; 1984b). This observation suggests that the coronavirus DI particles are unique since the DI genomes of other viruses usually exhibit more extensive deletions. Additionally, coronavirus DIs interfere with the replication of the standard virus to a much smaller extent. We have further shown that, with the exception of mRNA 7, the synthesis of the virus-specific mRNAs in DI-infected cells is inhibited. Instead, the infected cells contain two novel RNA species: one is the DI-specific genomic RNA (DIssA), which is eventually incorporated into the virus particles, the other novel RNA species is subgenomic but of variable size, depending on the level of DI passage. This subgenomic RNA is single-stranded, polyadenylated and contains sequences derived from various noncontiguous parts of the DI genome (Makino et al., 1985). In this report, we present data on the possible mechanisms of synthesis of the two DI-specific RNA species.

\section{MATERIALS AND METHODS}

Viruses and Cell Culture

MHV-JHM and MHV-A59 were used as nondefective standard viruses. The serially passaged MHV-JHM stock at passage level 17 was used as the source of DI particles (Makino et al., 1985). This virus stock inhibits the growth of 
standard MHV-JHM about 50\%-60\% (Makino et al., 1984a). All viruses were propagated in DBT cells, and viral RNA isolated as described previously (Makino et al., 1984a).

\section{Determination of ultraviolet light target size}

At four hour post-infection, the culture medium was replaced with Eagle's minimal essential medium (MEM) containing 5\% dialyzed fetal calf serum, $1 / 10$ normal concentration of phosphate and 2.5ug of actinomycin D per ml. After incubation for 3 hour, the medium was removed, and the cell monolayer was UV irradiated for various periods of time. A 30-W germicidal lamp at a distance of $50 \mathrm{~cm}$ was employed. After irradiation, the cultures were incubated for $60 \mathrm{~min}$ in phosphate-free MEM containing 5\% dialyzed fetal calf serum, 2.5ug actinomycin $\mathrm{D}$ and $250 \mathrm{uCi}$ of ${ }^{32} \mathrm{P}$-orthophosphate per $\mathrm{ml}$. Intracellular RNA was extracted after $60 \mathrm{~min}$ labeling and separated by agarose gel electrophoresis. The autoradiograph of the gel was analyzed by densitometry. The UV target size of each RNA species was determined by the expression In $(\mathrm{N} t / \mathrm{No})=-\mathrm{K} \times \mathrm{T} \times \mathrm{t}$, where $\mathrm{Nt}$ is the rate of RNA synthesis after $t$ seconds of irradiation, No is the RNA synthesis in the unirradiated culture, $T$ is the target size, and $K$ is a constant (Jacobs et al., 1981). Numerical values for the slopes of the curves obtained by linear regression analysis were used to calculate the target sizes for the individual RNAs (Jacobs et al., 1981).

Isolation of replicative form (RF) RNA and replicative intermediate (RI) RNA.

The intracellular RNA was dissolved in NTE buffer $(0.1 \mathrm{M} \mathrm{NaCl}, 0.01 \mathrm{M}$ Tris$\mathrm{HCl}$, $\mathrm{pH} 7.2$, and $1 \mathrm{mM}$ EDTA) and adjusted to $2 \mathrm{M} \mathrm{NaCl}$ and $0.05 \%$ SDS. The solution was left at $4^{\circ} \mathrm{C}$ for $24 \mathrm{hr}$ and centrifuged at 15,000 r.p.m. for $60 \mathrm{~min}$. The supernatant fraction was diluted and exhaustively digested with RNase A. The resulting RNase-resistant RNA represents double-stranded RF RNA (Baric et al., 1983). The $2 \mathrm{M} \mathrm{NaCl}$ precipitate was dissolved in water, precipitated with ethanol and then redissolved in NTE buffer containing 1\% SDS. The viral RNA was fractionated by Sepharose 2B-CL column chromatography as described (Baric et al., 1983). The RI RNA, which represents partially double-standed and partially single-stranded RNA structure, was present in the void volume fractions.

\section{RESULTS}

Lack of incorporation of DI subgenomic RNA into virus particles.

To determine the mechanism of DI RNA synthesis, we first examined whether the various species of DI-specific RNAs were incorporated in the virus particles. The intracellular RNA and the RNA isolated from the purified virion released from the same DI-infected cells were compared directly (Fig. 1). It is evident that only the DI-genomic RNA (DIssA) was incorporated in the DI virus particles. Neither the DIssE nor mRNA 7 were incorporated. However, we could not rule out the possibility that a minute amount of DIssE was packaged into virus particles. Indeed, a prolonged exposure of the autoradiogram indicated that the DI virus preparation contained a trace amount of mRNA 7 and DIssE (data not shown), suggesting that these subgenomic RNAs could be packaged nonspecifically. Nevertheless, this result suggests that DIssE is similar to the subgenomic mRNA, a transcription product, and not a replication product which can be efficiently packaged into virions.

Characterization of RF RNA and RI RNA in DI-infected cells.

It has previously been shown that all of the MHV mRNAs are synthesized from genome-sized RI RNA and that only one RF species is present in MHV- 


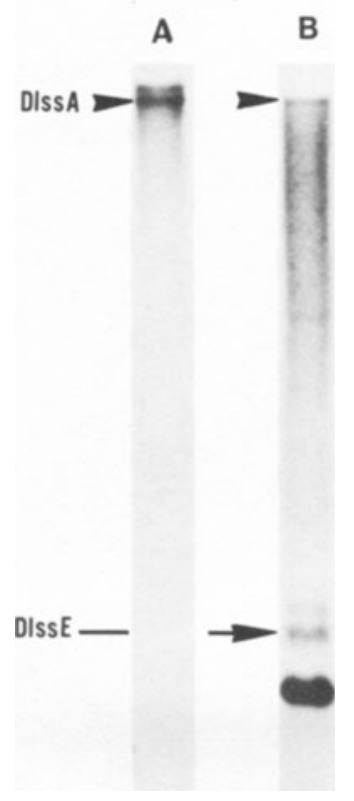

Fig. 1. Agarose gel electrophoresis of glyoxal-denatured ${ }^{32} \mathrm{P}$-labeled virion RNA (A) and intracellular RNAs (B) of DI at the same passage level.

infected cells (Baric et al., 1983). We thus studied whether there was a separate RI or RF for the DI-specific RNA species.

Electrophoresis of the double-stranded RF RNA from both JHM- and DIinfected cells revealed a single RNA band with an electrophoretic mobility similar to that of the genomic RNA (Fig. 2A). No subgenomic RF RNAs were detected in either of the RNA samples. Likewise, the partially double-stranded and partially single-stranded RI RNA from DI-infected cells also showed a single RNA band (Fig. 2B). This RNA migrated slightly faster than the RI of the standard JHM virus. Trace amounts of the standard JHM RI RNA were also detected in DI-infected cells. No subgenomic RI RNA was apparent. However, we could not rule out the possibility that a trace amount of subgenomic RI RNA might be present but obscured by a slight degradation of genomic-sized RNA. This result suggests that the DI-specific subgenomic RNA is likely synthesized from a larger, possibly genome-sized RNA template.

Dependence or independence of helper functions for the transcription of DIspecific RNAs

The oligonucleotide fingerprinting analysis showed that the DI genomic RNA contains multiple deletions as compared to the standard viral genome; however, the 5'-end gene A, which presumably encodes viral RNA polymerases, is essentially intact (Makino et al., 1985). This raises a possibility that the DI genomic RNA may encode a functional, although modified, polymerase. Thus DI RNA may be synthesized in the absence of helper functions provided by the standard virus. To test this possibility we examined the effects of virus dilution on the synthesis of DI-specific RNAs. Since the virus stock used in this experiment contained both DI particles and standard JHM virus, serial virus dilution would progressively reduce the possibility of co-infection of any single cell by both DI particles and standard viruses. Thus, if the DI RNA synthesis requires helper functions from the standard virus, the DI RNA synthesis should 


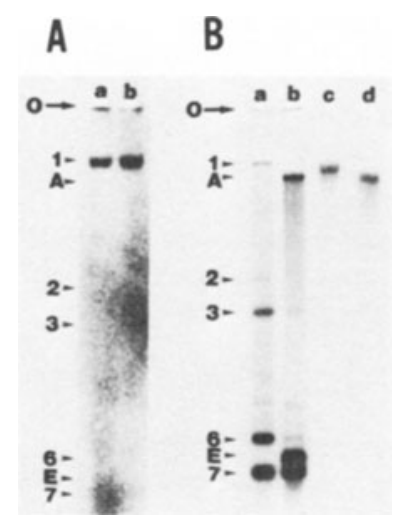

Fig. 2. Electrophoretic analysis of RF RNA (A) and RI RNA (B) isolated from MHV- $35 \mathrm{M}$ - and DI-infected cells.

(A): ${ }^{32}$ p-labeled double-stranded RF RNA of MHV-JHM-(a) or DI-(b) infected cells was electrophoresed on $1 \%$ agarose gels without denaturation.

(B): 32 p-labeled RI RNAs from MHV-JHM-(c) or DI-(d) infected cells. RNA samples were separated by agarose gel electrophoresis under nondenaturing conditions. Single-stranded poly (A)-containing RNAs from MHV-JHM-(a) and DI-(b) infected cells were included as controls. 0: Origin of the gels. Arrowheads denote the major MHV-JHM mRNAs (1,2,3,6 and 7) and DI-specific intracellular RNAs (A and E).

decrease exponentially following virus dilution, according to a two-hit kinetics. In contrast, a one-hit kinetics would indicate that DI RNA can be synthesized independently of helper functions.

The cells were infected with a serial dilution of DI-containing JHM stock and the amount of RNA synthesis determined. As shown in Fig. $3 \mathrm{~A}$ and 3B, the synthesis of DIssA, which is equivalent to DI genomic RNA, decreased linearly following virus dilution, according to a one-hit kinetics, suggesting that DIssA was synthesized in the absence of helper functions. Thus the DI genomic RNA contains all the genetic information required for DI RNA replication. In contrast, the reduction of DIssE RNA synthesis followed a two-hit kinetics, suggesting that the helper functions are required.

This interpretation was further tested by performing a virus dilution experiment in the presence of excess standard JHM virus (m. o. $\left.\mathrm{i}_{.}=5\right)$. As shown in Fig. 3C and 3D, the kinetics of DIssE RNA synthesis was converted to a linear regression, similar to that of DIssA. These studies demonstrated that the transcription of subgenomic DI RNA requires trans-acting products which are supplied by co-infecting standard virus.

\section{Structure of DIssE after co-infection with MHV-A59}

One possible helper function supplied by the standard virus for the synthesis of the DI subgenomic RNA is the sharing of the leader RNA from the standard virus, since the leader RNA has been shown to act in trans (Makino et al., 1986b). To examine this possibility, we studied the structure of DIssE RNA obtained from cells co-infected with MHV-A59 and DI particle. As shown in Fig. 4 , the oligonucleotide fingerprint of DIssE from this mixed infection is indistinguishable from that of DIssE obtained in a single infection. Both DIssE RNAs contained JHM-specific leader T -oligonucleotide 8 and other DIssEspecific $T_{1}$ spots (Makino et al., 1985). The leader-specific oligonucleotide of A59 was not detected in DIssE after co-infection with MHV-A59. This study suggests that the helper function required for DIssE synthesis could not be supplied by A59 leader RNA. 

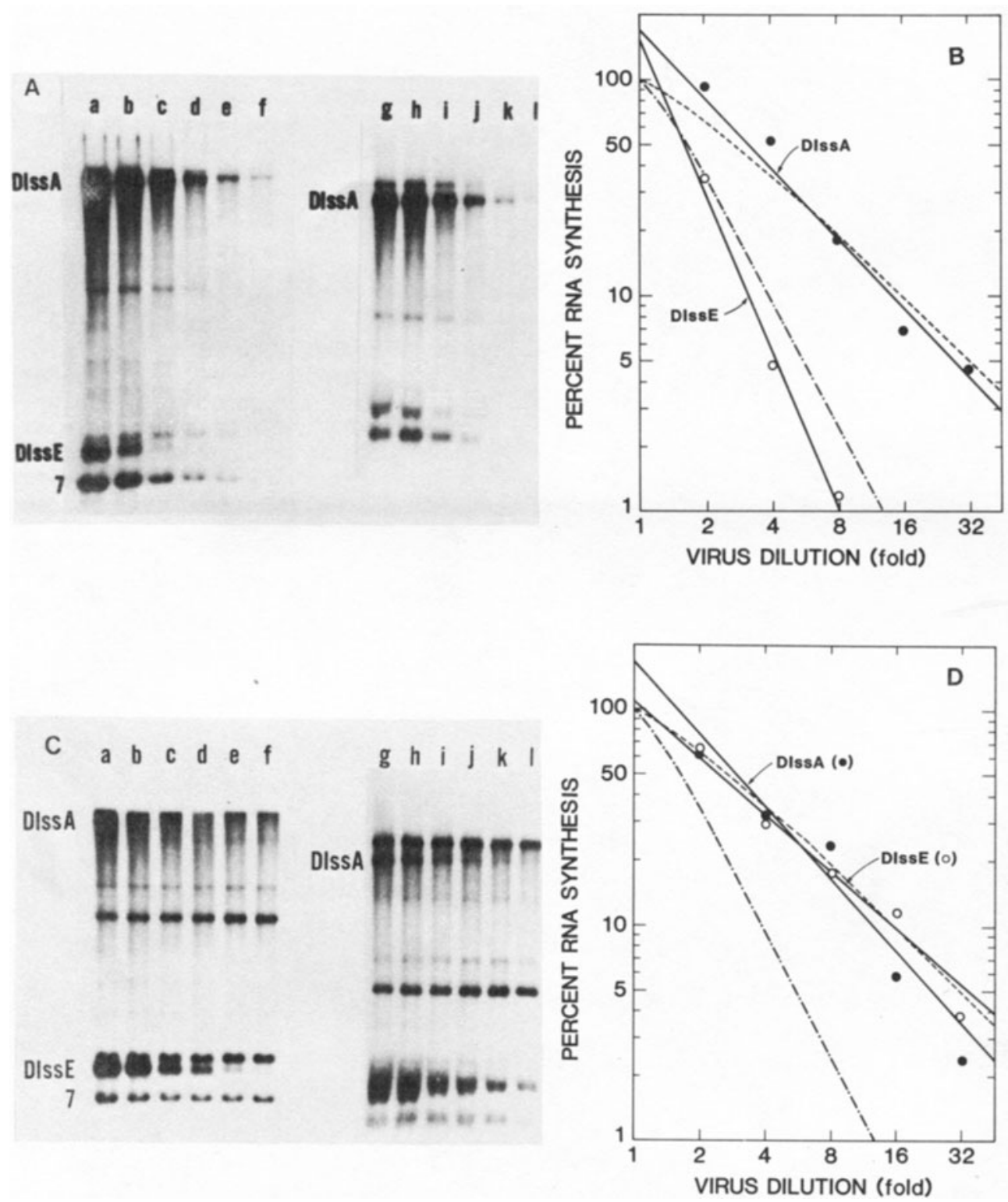

Fig. 3. Effects of helper virus infection on DI RNA synthesis.

(A,C): DBT cells were infected with either two-fold serially diluted DIcontaining virus (A) or a mixture of şerially diluted DI virus and an excess amount of standard MHV-JHM(C). $\quad{ }^{2}$ p-labeled virus-specific RNAs were separated by electrophoresis following glyoxal treatment (a-f) or without glyoxal $(\mathrm{g}-\mathrm{l})$. The lanes $\mathrm{a}$ and $\mathrm{g}$ are undiluted viruses at m.o.i. of 1 . The remaining lanes are two-fold serially diluted. (B,D): The autoradiographs in $A$ and $C$ were quantified by densitometry and the graphs were fitted by linear regression analysis. Theoretical one-hit kinetics (-----) and two-hit kinetics (-- - ) curves are also shown. B and D correspond to $A$ and $C$, respectively. 

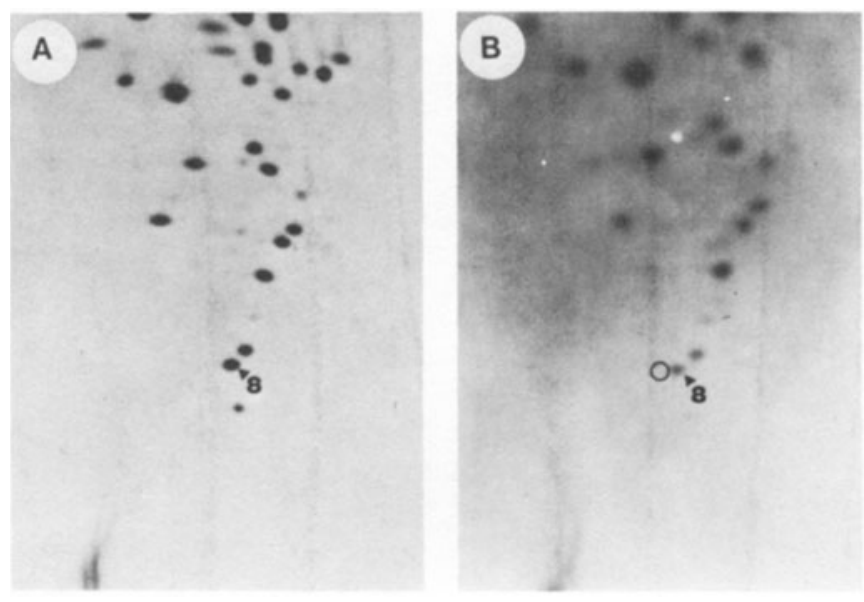

Fig. 4. Oligonucleotide fingerprints of DIssE RNA from the cells infected with

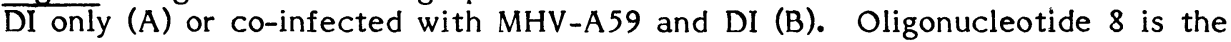
leader-specific oligonucleotide of DIssE. The circle represents the predicted location of MHV-A 59 leader-specific oligonucleotide 10.
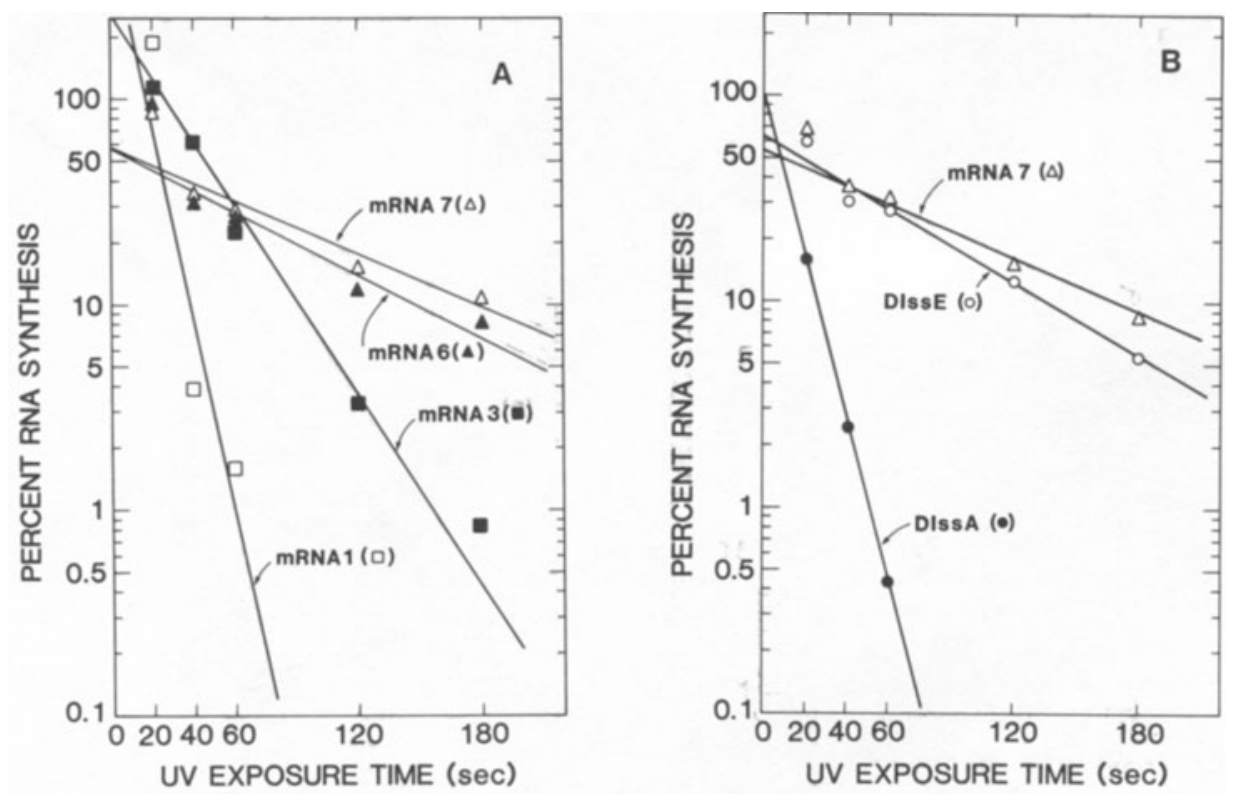

Fig. 5. Kinetics of inactivation by UV irradiation of MHV-JHM mRNA synthesis (A) and DI RNA synthesis (B). 
To further discern the mechanism of DI RNA synthesis, we examined the UV target size for the synthesis of DI-specific intracellular RNAs. This study was carried out to determine the size of RNA template for the synthesis of various DI RNA species.

DI-infected cells were irradiated with UV light at $7 \mathrm{hr}$ p.i., when the (+)strand RNA synthesis is at its peak. The amount of RNA synthesis was determined after various doses of UV irradiation. The kinetics of inhibition of RNA synthesis relative to the dose of UV irradiation is shown in Fig. 5. The UV target sizes for individual RNA species were determined (Table 1). It is evident that the target sizes of DI-specific RNAs are nearly identical to their respective physical sizes. These data are consistent with the models that subgenomic DI RNAs are transcribed either from a template of their own size or from the genomic RNA template by a process of jumping transcription.

Table 1. UV target sizes of various RNA species

\begin{tabular}{llll}
\hline Exp RNA & KxT (S $\left.{ }^{-1}\right)^{\mathrm{a}}$ & $\begin{array}{l}\text { Estimated target } \\
\text { Size }\left(\mathrm{M} . \mathrm{W} \cdot \times 10^{-6}\right)\end{array}$ & $\begin{array}{l}\text { Physical size } \\
\text { of RNA }\left(\mathrm{M} . \mathrm{W} \cdot \times 10^{-6}\right)\end{array}$
\end{tabular}

$\begin{array}{lllll} & & & & \\ \text { ARNA 7 } & 9.91 \times 10^{-3} & 0.60 & 0.60 \\ \text { A } & \text { mRNA 6 } & 1.31 \times 10^{-2} & 0.79 & 0.86 \\ & \text { mRNA 3 } & 3.53 \times 10^{-2} & 2.14 & 2.60 \\ & \text { mRNA 1 } & 1.09 \times 10^{-1} & 6.60 & 5.40 \\ & & & & \\ & & & 0.60 & 0.60 \\ \text { BRNA 7 } & 9.17 \times 10^{-3} & 0.86 & 0.78 \\ \text { B DIssE } & 1.32 \times 10^{-2} & 5.84 & 5.20\end{array}$

$\mathrm{a}_{\mathrm{K} x \mathrm{~T}}$ was calculated from the equation $\ln (\mathrm{Nt} / \mathrm{No})=-\mathrm{K} \times \mathrm{T} x \mathrm{t}$, where $\mathrm{Nt}$ represents the remaining synthesis of RNA after $t$ seconds of UV irradiation; No is theRNA synthesis in unirradiated culture. $\mathrm{T}$ is the target size; and $\mathrm{K}$ is a constant. The calculation was made from data illustrated in Fig. 5 by using linear regression analysis.

${ }^{b}$ The molecular weights of RNAs were obtained according to the data of Makino et al (1984b, 1985).

\section{DISCUSSION}

The data presented in this report suggested two contrasting mechanisms of synthesis for DI-specific-RNA species. On the one hand, DI genomic sized RNA (DIssA) appears to contain only a small degree of deletions and is able to replicate by itself. It may encode its own polymerase and have a template RNA of corresponding size. On the other hand, the subgenomic DIssE RNA appears not to be incorporated into virion particles, does not have a template RNA of its own size and requires helper function(s) for synthesis, but its UV target size is the same as its physical size. Two possible models are compatible with these data: one model is that the subgenomic DI RNAs are transcribed from the fulllength DI genomic RNA by a mechanism of discontinuous, jumping transcription. In other words, RNA transcription could stop at certain termination signals and resume at downstream initiation sites. This is consistent with the standard coronavirus mRNA transcription, which has been shown to utilize a discontinuous mechanism involving a free leader RNA species (Baric et al., 1985). DI RNA 
could have different termination and initiation signals; as a result, RNA transcription skips several parts of the genomic template, generating a DIssE RNA with discontiguous stretches of viral sequences. The second possibility is that a very small amount of DI subgenomic RNAs is packaged within the virus particle. By virtue of its small size, it could be replicated at a much faster rate by the RNA polymerase encoded from helper viruses. We have found that a trace amount of mRNA 7 and DI subgenomic RNA was co-purified with the DI virus particle. Since both RNA species were present in the same amount, they most likely represent contaminants, or were packaged accidentally. It is not clear whether this DI subgenomic RNA could serve as the template for subsequent replication. In any case, the DI subgenomic RNAs appear to be very poorly, if at all, packaged. Since both the DI genomic and subgenomic RNAs contain 5'-end genomic sequences, which presumably contain packaging signals, the comparison of these RNAs sequences are likely to reveal features required for encapsidation.

The finding that the replication of DI genome-sized RNA did not require any helper function suggests that DI RNA encodes functional RNA polymerases. This result is consistent with our previous oligonucleotide fingerprinting sturfies which show that the gene A, encoding RNA polymerase, is essentially intaci in the DI genome. Only a minor difference was detected in this region between DI and standard JHM genome (Makino et al, 1985). It is possible that this altered polymerase, in association with other helper functions, is responsible for the subgenomic DI RNA transcription. Another consequence of this finding is that it might be possible to isolate a persistently infected cell line in which only DI genome is transcribed. Such a cell line will be very useful for understanding the mechanism of persistent viral infection.

Although DI genomic RNA is not defective in replication, it is defective in several regions of genome as shown by multiple changes of oligonucleotide fingerprints (Makino et al., 1985). It would be interesting to know whether this RNA alone interferes with the replication of the standard viral RNA. In contrast, the subgenomic DI RNAs are more similar to the DI RNA of other animal viruses. These subgenomic mRNAs may be the RNA species which interfere with the transcription and replication of standard virus. However, this small RNA is poorly incorporated in the virus particle. Thus, coronavirus DI is a very unique system. It will be very interesting to examine the structural and biological relationship between the various DI-specific RNA species.

\section{ACKNOWLEDGMENTS}

We would like to thank Carol Flores for excellent typing and Drs. Lisa Soe and Susan Baker for helpful comments. This work was supported in part by U.S. Public Health Research Grants AI 19244 and NS 18146 and National Multiple Sclerosis Society Research Grant RG 1449.

\section{REFERENCES}

Baric, R.S., Stohlman, S.A., and Lai, M.M.C. (1983). Characterization of replicative intermediate RNA of mouse hepatitis virus: Presence of leader RNA sequences on nascent chains. J. Virol. 48, 633-640.

Baric, R.S., Stohlman, S.A., Razavi, M.K., and Lai, M.M.C. (1985). Characterization of leader-related small RNAs in coronavirus-infected cells: Further evidence for leader-primed mechanism of transcription. Virus Res. 3, 19-33. 
Jacobs, L., Spaan, W.J.M., Horzinek, M.C. and van der Zeijst, B.A.M. (1981). Synthesis of subgenomic mRNA's of mouse hepatitis virus is initiated independently: Evidence from UV transcription mapping. J. Virol. 39, 401-406.

Makino, S., Fujioka, N., and Fujiwara, K. (1985). Structure of the intracellular defective viral RNAs of defective interfering particles of mouse hepatitis virus. J. Virol. 54, 329-336.

Makino, S., Stohlman, S.A. and Lai, M.M.C. (1986). Leader sequences of coronavirus mRNAs can be freely reassorted: Evidence for the role of free leader RNA in transcription. Proc. Natl. Acad. Sci. U.S.A. 83, 4204-4208.

Makino, S., Taguchi, F., and Fujiwara, K. (1984a). Defective interfering particles of mouse hepatitis virus. Virology 133, 9-17.

Makino, S., Taguchi, F., Hirano, N. and Fujiwara, K. (1984b). Analysis of genomic and intracellular viral RNAs of small plaque mutants of mouse hepatitis virus, JHM strain. Virology 139, 138-151. 